\title{
SAGES guidelines for laparoscopic peritoneal dialysis access surgery
}

\author{
John H. Crabtree
}

Received: 15 July 2014/Accepted: 8 August 2014/Published online: 23 August 2014

(C) Society of American Gastrointestinal and Endoscopic Surgeons (SAGES) 2014

The percentage of peritoneal dialysis (PD) catheters implanted laparoscopically in the USA has almost doubled from $26 \%$ in 2007 to nearly $50 \%$ in 2012 [1]. With this dramatic change in surgical practice, the Society of American Gastrointestinal and Endoscopic Surgeons (SAGES) has undertaken the enormous task of formulating guidelines for laparoscopic PD access surgery. Since the laparoscopic approach for PD access is still evolving with almost as many versions of the technique as there are surgeons performing them, SAGES is to be commended for their effort to bring order and guiding principles to this method of catheter placement.

The essential point that surgeons should take away from the Guidelines is that when the laparoscope is used only to witness the position of the catheter tip, the outcomes are no different than any other catheter insertion method. Simply using the laparoscope to locate the catheter is blatant underutilization of this modality and should be viewed as unacceptable practice. The advantages of laparoscopy over every other technique are the adjunctive procedures enabled by this method, principally rectus sheath tunneling, omentopexy, adhesiolysis, epiploectomy, salpingectomy, and colopexy. When these techniques are applied effectively, the laparoscopic approach can both prevent and resolve most of the common mechanical problems that complicate insertion of PD catheters.

A few of the Guidelines merit further emphasis or require a counter viewpoint.

\section{J. H. Crabtree $(\square)$}

Society for Education and Research in Peritoneal Access Surgery, 340 South Lemon Avenue, Suite 2404, Walnut, CA 91789, USA

e-mail: johncrabtree@sbcglobal.net
Guideline 11 Tunneling of the catheter in a craniocaudal direction through the rectus sheath toward the pelvis has been variously described as "rectus sheath tunneling," "musculofascial tunneling," "extraperitoneal tunneling," and "preperitoneal tunneling" [2-5]. Importantly, the SAGES Guidelines standardize the terminology by promoting a word description that correctly reflects the anatomic pathway of the tunneling process, therefore, rectus sheath tunneling. This enables surgeons to have a clear understanding of the concept. Tunneling begins within the rectus sheath on the surface of the posterior rectus sheath. This is the exact same plane used to insert the structural balloon trocar for total extraperitoneal hernia repair. For PD catheter insertion, approximately two-thirds to threefourths of the tunnel is within the rectus sheath. At the arcuate line, the tunneling process perforates the transversalis fascia which below the arcuate line rests directly against the posterior aspect of the rectus muscle. At this point, the tunneling process enters into the preperitoneal space. Only a short length of the tunnel is preperitoneal before entry is made into the peritoneal cavity. The length of the tunnel is limited by the span of available port devices.

Rectus sheath tunneling effectively keeps the catheter oriented toward the pelvis, eliminates pericatheter hernias, and reduces the risk of pericatheter leaks. Other than the laparoscope port, it does not require additional working ports or suturing. Currently, it is best performed with a step-port device (7/8 mm Mini Step, Covidien, Mansfield, MA, USA), permitting the small caliber Veress needle with overlying expandable sleeve to be steered precisely down the rectus sheath with minimal tissue plane disruption and avoidance of the inferior epigastric vessels. In the event of unavailability of the Mini Step instrument, recommended port specifications for rectus sheath tunneling include a 
bladeless device, 7-8 $\mathrm{mm}$ internal working diameter, smooth cannula (no threads), and working length $\geq 100 \mathrm{~mm}$. For non Mini Step devices, a hook cautery can be passed through the port at the lower end of the tunnel to create a safe peritoneal entry point under laparoscopic vision. Rectus sheath tunneling with available port devices depart from their intended use. Clearly, there is a need to develop a dedicated apparatus specifically designed to insert PD catheters through a long rectus sheath tunnel in a simple, safe, accurate, and reproducible fashion while maintaining pneumatic competence and visibility during laparoscopy.

Rectus sheath tunneling obviates the need for suturing the catheter tip to a pelvic structure. In addition to requiring extra ports and time to perform, 6-9 \% of pelvic sutures erode from the tissues during the short-term resulting in an unacceptable incidence of catheter tip migration [6, 7]. On occasion, it has been reported that the anchoring suture held and required a laparoscopic procedure to cut the stitch to permit catheter removal [8].

Guidelines 12-13 Omentopexy should be the preferred procedure for avoiding omental entrapment of the catheter tip from the standpoint of its simplicity, reduced operative time and cost, and avoidance of the postoperative risk of hemorrhage that accompanies omentectomy. Omentopexy should be employed selectively since it may be unnecessary when omentum is short or adherent to previous upper abdominal surgical sites. There is no evidence that omentopexy would be ineffective in the pediatric population-it just appears not to have been yet tried. An additional argument against omentectomy is that omental milky spots (clusters of leukocytes) appear to have a role in the peritoneal cavity immune response, especially in the pediatric population [9].

Guideline 19 Many laparoscopic surgeons remain tied to the umbilicus and appear hopelessly unable to detach themselves from this cicatricial birthmark for Veress needle and laparoscope placement. Periumbilical midline port sites are prone to develop hernias in PD patients due to the elevated intraperitoneal hydrostatic pressure associated with dialysis solution. Port site hernias in PD patients have a reported incidence of 3-9 \% [6, 10-13]. Furthermore, the conventional periumbilical region for laparoscope placement is too close to the catheter insertion site to be practical. This close proximity results in port conflict, poor visibility, and frustration with the procedure. For optimal visualization and ergometric use of laparoscopic instruments, the laparoscope and accessory ports should be placed 16-18 cm from the target area [14]. Fortunately, the increased experience with non-PD-related laparoscopic ventral and incisional hernia repairs has boosted the surgeon's confidence with initial Veress and port placement off of the midline.
Guideline 16 The surgical community currently views mechanical bowel preps with skepticism. Clinical trials reveal that mechanical bowel prep prior to colorectal surgery does not lower the complication rate; however, this evidence should not be extrapolated to peritoneal access surgery. While mechanical bowel prep for colorectal surgery was aimed at lessening the risk of anastomotic leak, infection, and mortality, peritoneal access surgery is concerned with avoiding catheter flow complications from extrinsic compression of catheter side drainage holes or displacement of the catheter tip to a position of poor drainage function by distended fecal-filled bowel. Constipation is a common problem among chronic kidney disease patients. Postoperative narcotic analgesic requirements further aggravate this underlying propensity for constipation. Any experienced PD practitioner will affirm that constipation is the most common reason for post procedure catheter flow dysfunction, having been noted in $68 \%$ of patients with constipation at first dialysis, compared to only $13 \%$ in patients without constipation [15]. Moreover, the necessity of acute treatment of postoperative constipation may lead to transmural migration of bacteria resulting in peritonitis [16].

Patients should be questioned about constipation symptoms during the initial consultation and started on stool softeners if this has not already been prescribed. An effective protocol for mechanical bowel prep before catheter implantation utilizes polyethylene glycol solution, having the patient consume $2 \mathrm{~L}$ the day prior to the procedure or $1 \mathrm{~L}$ /day for 2 days before surgery.

Guideline 33 Contrary to the Guideline statement, pain during PD is not rare, especially drain pain. Clinically significant drain pain has a reported incidence of 13-25\% of patients [17, 18]. Drain pain occurs when visceral structures siphon up to the catheter tip and impact it against the exquisitely sensitive parietal peritoneum. The phenomenon occurs more frequently with cycler PD where hydraulic suction rather than gravity is used to drain the dialysate. Drain pain is more likely to occur when the catheter tip is implanted too low in the pelvis, wedging it between the rectum and uterus or rectum and bladder, leaving it susceptible to early termination of dialysate outflow and abrupt contact of the catheter tip with the peritoneum. In similar fashion, catheter tip malposition can diminish outflow and produce pain. Pain from excessively low placement of the catheter tip in the pelvis that is not improved by tidal PD (leaving a residual volume of dialysate above the point where pain occurs) or by conversion to non-cycler PD using gravity-only drainage may lead to catheter removal, often with the patient abandoning PD altogether. There is no satisfactory surgical salvage procedure short of replacement for a catheter implanted too deep in the pelvis. Prevention of the problem can be 
achieved by employing methodology during preoperative planning that uses the catheter itself to determine the insertion site that produces proper pelvic position of the catheter tip [19]. Alternatively, some catheter manufacturers provide marking stencils for their catheters that embody specific device dimensions and anatomic references to identify the appropriate insertion site (MedComp, Inc., Harleysville, PA, USA; Merit Medical, South Jordan, UT, USA).

Accrual of evidence of sufficient quality to compel a consensus of procedural standards for laparoscopic peritoneal access has been slow to come about. The number of catheter placements performed by individual surgeons or medical groups is usually small; therefore, studies are frequently underpowered and unable to deliver convincing results. This state of affairs accounts for the fact that 22 of the 36 Guidelines are based upon low or very low grade evidence and/or weak recommendations. Favorably, the PD Outcomes and Practice Patterns Study (PDOPPS) were launched in late 2013 [20]. Modeled upon the success of the Dialysis Outcomes and Practice Pattern Study (DOPPS), PDOPPS is designed to conduct multicenter and multi-country studies on optimal practice. One of the studies, Optimizing Early Dialysis Catheter Function, may assist in refining future SAGES guidelines for laparoscopic PD access surgery.

Disclosures John H. Crabtree is a consultant for MedComp, Inc. and Baxter Healthcare. He is a member of the speaker's bureau for Baxter Home Therapies Institute, DaVita Healthcare Partners, and Fresenius Medical Care Advanced Renal Education Program.

\section{References}

1. Physician/Supplier Procedure Summary Master File, 2007-2012. Baltimore, MD: Centers for Medicare \& Medicaid Services, Department of Health \& Human Services

2. Crabtree JH, Burchette RJ (2009) Effective use of laparoscopy for long-term peritoneal dialysis access. Am J Surg 198:135-141

3. Rui M, Figueiredo N, Costa P (2008) Laparoscopic placement of Tenckhoff catheters for peritoneal dialysis: a safe, effective, and reproducible procedure. Perit Dial Int 28:170-173

4. Ogunc G (2005) Minilaparoscopic extraperitoneal tunneling with omentopexy: a new technique for CAPD catheter placement. Perit Dial Int 25:551-555

5. Comert M, Borazan A, Kulah E, Ucan BH (2005) A new laparoscopic technique for the placement of a permanent peritoneal dialysis catheter. Surg Endosc 19:245-248
6. Bar-Zohar D, Sagie B, Lubezky N, Blum M, Klausner J, AbuAbeid S (2006) Laparoscopic implantation of the Tenckhoff catheter for the treatment of end-stage renal failure and congestive heart failure: experience with the pelvic fixation stitch. IMAJ 8:174-178

7. Gunes ME, Uzum G, Koc O, Duzkoylu Y, Kucukyilmaz M, Sari YS, Tunali V, Kose S (2013) A modified method in laparoscopic peritoneal catheter implantation: the combination of preperitoneal tunneling and pelvic fixation. ISRN Surg. doi:10.1155/2013/ 248126

8. Santarelli S, Zeiler M, Marinelli R, Monteburini T, Federico A, Ceraudo E (2006) Videolaparoscopy as rescue therapy and placement of peritoneal dialysis catheters: a thirty-two case single centre experience. Nephrol Dial Transplant 21:1348-1354

9. Beelen RH, Oosterling SJ, van Egmond M, van den Born J, Zareie M (2005) Omental milky spots in peritoneal pathophysiology (spots before your eyes). Perit Dial Int 25:30-32

10. Lu CT, Watson DI, Elias TJ, Faull RJ, Clarksont AR, Bannister KM (2003) Laparoscopic placement of peritoneal dialysis catheters: 7 years experience. ANZ J Surg 73:109-111

11. Soontrapornchai P, Simapatanapong T (2005) Comparison of open and laparoscopic secure placement of peritoneal dialysis catheters. Surg Endosc 19:137-139

12. Haggerty SP, Zeni TM, Carder M, Frantzides C (2007) Laparoscopic peritoneal dialysis catheter insertion using a Quinton percutaneous insertion kit. JSLS 11:208-214

13. Schmidt SC, Pohle C, Langrehr JM, Schumacher G, Jacob D, Neuhaus P (2007) Laparoscopic-assisted placement of peritoneal dialysis catheters: implantation technique and results. J Laparoendosc Adv Surg Tech A 17:596-599

14. Ferzli GS, Fingerhut A (2004) Trocar placement for laparoscopic abdominal procedures: a simple standardized method. J Am Coll Surg 198:163-173

15. Vijt D, Castro MJ, Endall G, Lindley E, Elseviers M (2004) Post insertion catheter care in peritoneal dialysis (PD) centres across Europe-Part 2: complication rates and individual patient outcomes. EDTNA ERCA J 30:91-96

16. Singharetnam W, Holley JL (1996) Acute treatment of constipation may lead to transmural migration of bacteria resulting in gram-negative, polymicrobial, or fungal peritonitis. Perit Dial Int $16: 423-425$

17. Juergensen PH, Murphy AL, Pherson KA, Chorney WS, Kliger AS, Finkelstein FO (1999) Tidal peritoneal dialysis to achieve comfort in chronic peritoneal dialysis patients. Adv Perit Dial 15:125-126

18. Blake PG, Sloand JA, McMurray S, Jain AK, Matthews S (2014) A multicenter survey of why and how tidal peritoneal dialysis (TPD) is being used. Perit Dial Int 34:458-460

19. Crabtree JH (2003) Construction and use of stencils in planning for peritoneal dialysis catheter implantation. Perit Dial Int 23:395-398

20. Perl J, Robinson B, Davies S (2014) Update on the Peritoneal Dialysis Outcomes and Practice Patterns Study (PDOPPS). Perit Dial Int 34:332 